\title{
Skin tightening by an insulated microneedle radiofrequency device combined with lower blepharoplasty: a randomized fellow eye comparison study
}

\author{
Preamjit Saonanon, $\mathrm{MD}^{1} \mathbb{D}$, Krit Rattanakit, $\mathrm{MD}^{2} \mathbb{D}$, Patnapa Vejanurug, $\mathrm{MD}^{3}$ (D), Apichaya Thanyavuthi, $\mathrm{MD}^{4}$ (iD \\ 'Department of Ophthalmology, King Chulalongkorn Memorial Hospital, Faculty of Medicine, Chulalongkorn University, Bangkok, Thailand \\ ${ }^{2}$ Division of Facial Plastic and Reconstructive Surgery, Department of Otolaryngology, Faculty of Medicine, Chulalongkorn University, Bangkok, \\ Thailand \\ ${ }^{3}$ Institute of Dermatology, Department of Medical Services, Ministry of Public Health, Bangkok, Thailand \\ ${ }^{4}$ Department of Dermatology, Siriraj Hospital, Faculty of Medicine, Mahidol University, Bangkok, Thailand
}

Background: Aging in the lower eyelids creates bags under the eyes from the protruding orbital fat, and periorbital wrinkles. Therefore, lower blepharoplasty should be combined with a skin tightening procedure to enhance the results.

Objective: To compare the effects between lower blepharoplasty combined with an insulated microneedle radiofrequency (RF) device and lower blepharoplasty alone.

Methods: Twelve Asian (24 eyes) patients with baggy eyelids were included. One eye per patient was randomized for lower blepharoplasty combined with insulated microneedle RF devices (RF group), and the other eye for lower blepharoplasty alone (LB group). All patients underwent transconjunctival lower blepharoplasty with fat transposition and orbicularis oculi suspension. The primary outcome was the difference between both groups on the five-point Wrinkle Assessment Scale (WAS), assessed at baseline and at 2 months post-surgery. The secondary outcome was patient satisfaction using the Global Aesthetic Improvement Scale (GAIS). Results: The WAS improvements were $75.0 \%$ (9/12 eyes) and 58.3\% (7/12 eyes) in the RF and LB groups, respectively. The GAIS showed high patient satisfaction in both groups, with an average of $4.12(3-5)$ in the RF group and $4.0(2-5)$ in the LB group ( $p=0.157)$. There was no difference in the postoperative inflammation between both eyes at 1 week; and no decrease in the WAS score at 2 months or any adverse effects at 6 months were observed.

Conclusion: Wrinkle improvement was observed post-lower blepharoplasty with or without microneedle RF treatment. The insulated microneedle RF device proved safe when performed concurrently with lower blepharoplasty.

Keywords: blepharoplasty; cosmetic techniques; needles; radiofrequency therapy; randomized controlled trial; skin aging

\section{Introduction}

The aging of the periorbital area occurs in every layer of tissue, including the skin, subcutaneous tissue, orbicularis oculi muscle, orbital fat, orbital ligaments, and orbital bone. The bony part undergoes resorption and the soft tissue increases in laxity as people age. Aging in the midface area manifests as a bag-like structure from protruding orbital fat and laxity of the periorbital soft tissue [1]. In the past, lower eyelid rejuvenation was achieved by means of orbital fat removal only. It has been

Received March 23, 2021; Revised April 14, 2021; Accepted April 16, 2021

Corresponding author: Krit Rattanakit

E-mail: crazykittyk@gmail.com

This is an Open Access article distributed under the terms of the Creative Commons Attribution Non-Commercial License (http://creativecommons.org/licenses/by-nc/4.0), which permits unrestricted non-commercial use, distribution, and reproduction in any medium, provided the original work is properly cited.

Copyright (C) 2021 Korean Society of Korean Cosmetic Surgery and Medicine (KSKCS \& KCCS). 
demonstrated that not only protrusion of orbital fat, but also descended malar fat pad and the presence of orbitomalar ligament and zygomatico-cutaneous ligament entrapment occur in midface aging, causing a double convexity deformity. Consequently, orbital fat removal alone does not provide satisfactory results. Current lower blepharoplasty techniques include the integration of orbital fat resection, release of the orbitomalar ligament, fat transposition, and horizontal lid tightening procedures [2]. Lower lid rejuvenation can be further enhanced by volume augmentation and skin rejuvenation $[1,2]$.

For skin rejuvenation, many surgeons perform lower blepharoplasty concomitantly with a fractional $\mathrm{CO}_{2}$ resurfacing laser [3]. Although fractional $\mathrm{CO}_{2}$ resurfacing lasers are very effective in periorbital wrinkle reduction, the main disadvantages include a long recovery period (2-4 weeks) and high risk of postinflammatory hyperpigmentation (PIH), especially in darker skin types [4]. In recent years, one of the newer technologies for skin tightening and wrinkle reduction has been the insulated microneedle radiofrequency ( $\mathrm{RF}$ ) device [5]. It has proved to be effective for periorbital wrinkle treatment. The applicator tip is $0.8 \mathrm{~mm}$ long, which is suitable for the skin thickness in the lower eyelid area [6]. The tip is partly insulated in order to protect the epidermis from thermal burn. When activated, RF energy is released that promotes collagen formation and remodeling, leading to a skin tightening effect. The microneedle RF device does not create a wound at the epithelial layer; therefore, it has a shorter recovery time of less than 1 week and a lower rate of $\mathrm{PIH}$ when compared to $\mathrm{CO}_{2}$ resurfacing lasers [6,7]. Utilizing a microneedle RF device as an adjuvant therapy in lower blepharoplasty is a novel method for optimizing the results of lower eyelid rejuvenation.

The aim of this prospective randomized fellow eye comparison study was to compare the surgical results of lower blepharoplasty alone with those of lower blepharoplasty performed simultaneously with a microneedle RF device, in terms of wrinkle reduction and patient satisfaction.

\section{Materials and methods}

Twelve patients from the Ophthalmic Plastic and Reconstructive Surgery unit of the Ophthalmology Department were included in the study from August 1, 2016 to January 28, 2017. The patients were aged 40-70 years, diagnosed with baggy eyelids, and willing to undergo lower blepharoplasty. None of the patients had undergone periorbital wrinkle treatment in the past 6 months. The exclusion criteria included: a history of previous lower blepharoplasty; botulinum toxin, filler, or fat injection to the periorbital area; and patients with lower lid laxity requiring a horizontal tightening procedure or with skin redundancy that required a skin removal procedure. The sample size was calculated using an algorithm based on a dependent sample and non-inferiority trial with a non-inferiority margin of 1 . This research adhered to the tenets of the Declaration of Helsinki, and was approved by the Institutional Review Board of Chulalongkorn University prior to its commencement (IRB no. 289/59).

After informed consent was obtained, the eyelid examinations were performed and digital photographs were taken. The patient characteristic data collected included age, sex, and Fitzpatrick skin type. All patients underwent bilateral transconjunctival lower blepharoplasty with fat transposition and orbicularis oculi suspension, which was performed by one oculoplastic surgeon (SP). Using a random number, one eye of each patient was randomized for insulated microneedle RF device application at the conclusion of the surgery (RF group) and the other eye for lower blepharoplasty alone (LB group). The randomization sequence was concealed in an opaque, sealed envelope sequentially numbered by the coordinator (KR), and was disclosed at the conclusion of the surgery.

All patients underwent the procedure with a local anesthesia solution of $2 \%$ lidocaine with 1:80,000 epinephrine. Bilateral transconjunctival lower blepharoplasty with fat transposition and orbicularis oculi suspension was performed and properly adjusted for each patient. Triangular-shaped redundant skin at the lateral canthus was also trimmed off after the orbicularis oculi suspension. The operation was performed first on the right eye and then on the left eye in all of all patients. In the RF group eye, the insulated microneedle RF (AGNES; GWSS cosmetic,

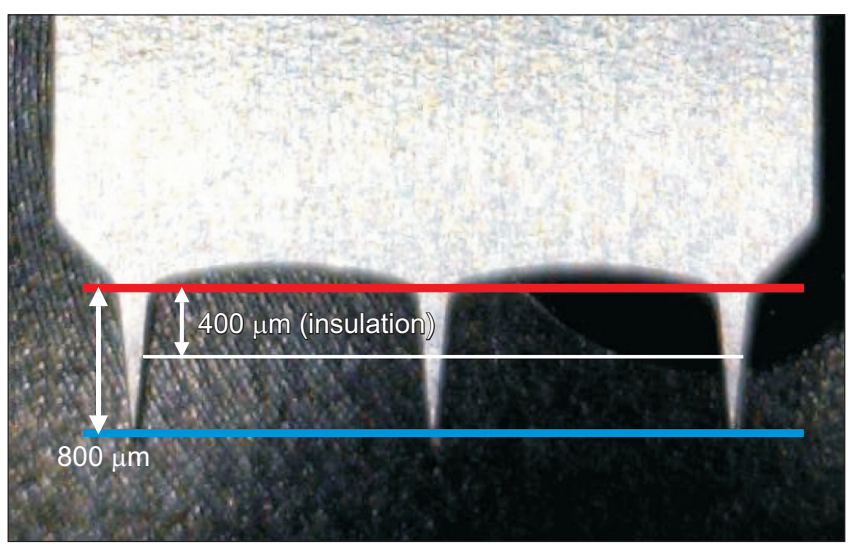

Fig. 1. The three-needle partly insulated apllicator tip of microneedle radiofrequency (AGNES). 
Seongnam, Korea) was applied to the lower eyelid skin using a three-needle partly-insulated applicator tip that penetrated $0.8 \mathrm{~mm}$ (Fig. 1, 2). The treatment parameters were 50 shots of penetration (Fig. 3) in one session using the monopolar mode of RF, level 3, power $4 \mathrm{~W}$, with a duration of $120 \mathrm{~ms}$. After applying the RF treatment to one eye, both lower eyelid areas were cooled with ice packs for 20 minutes. Patients were advised to use broad-spectrum sunscreen and avoid sun exposure. Two months after the operation, patients were allowed to voluntarily choose to receive microneedle RF treatment on the other eye that had not been treated intraoperatively (LB group eye).

\section{Outcome assessment}

High-resolution digital photographs were obtained at baseline and at 1 week, 1 month, 2 months, and 6 months after the operation using a Canon DSLR camera (Canon Inc., Tokyo, Japan) with identical camera settings, lighting, and patient posi-

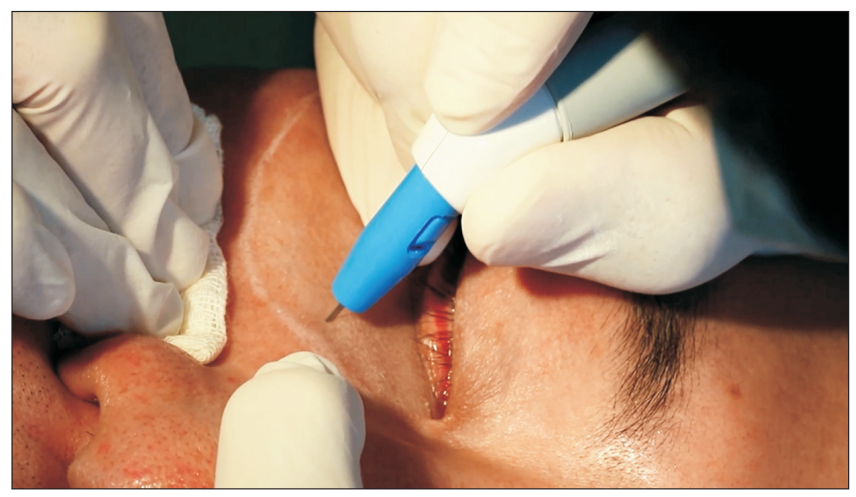

Fig. 2. Application of microneedle radiofrequency device (AGNES) at lower eyelid area.

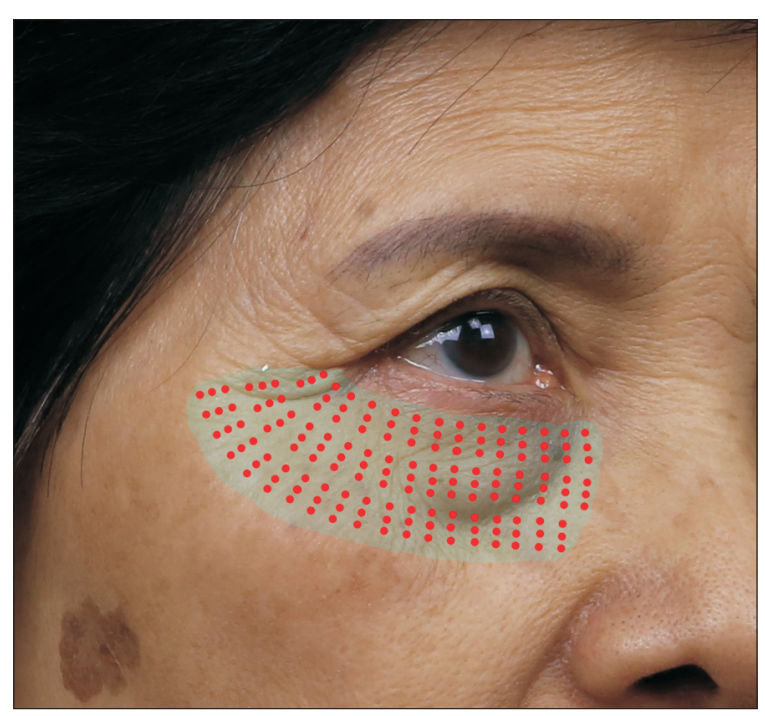

Fig. 3. Lower eyelid area for microneedle radiofrequency treatment. tioning. Preoperative and 2-month postoperative standardized photos of the patients were scored for wrinkle reduction by two dermatologists. The lower eyelid area of each eye was graded separately using the five-point Wrinkle Assessment Scale (WAS), which ranges from 0-4 (Table 1). Subject self-evaluation using the Global Aesthetic Improvement Scale (GAIS; Table 2) was also obtained 2 months postoperatively. Complications recorded included skin erythema, edema, erosion, hypopigmentation, $\mathrm{PIH}$, and scarring. The primary outcome was the difference between pre-and postoperative WAS scores in the RF and LB groups. The secondary outcome was the difference in the GAIS scores between both groups.

Descriptive statistics were used to evaluate baseline characteristics, with mean and standard deviations used for quantitative variables, and counts and percentages for categorical variables. The Wilcoxon signed-rank test was used to test the improvement in the WAS and the difference in the GAIS scores between the RF and LB groups. Statistical significance was set at $\mathrm{p}<0.05$, and the Statistical Package for the Social Sciences (IBM SPSS ver. 22.0; IBM Corp., Armonk, NY, USA) was used for all of the statistical analyses.

\section{Results}

Twelve Asian patients (24 eyes) with mild-to-moderate wrinkles were analyzed. There were 11 females and 1 male with

Table 1. Five-point Wrinkle Assessment Scale (WAS)

\begin{tabular}{ll}
\hline \multicolumn{1}{c}{ Score } & \multicolumn{1}{c}{ Description } \\
\hline 0 (none) & $\begin{array}{l}\text { No wrinkles } \\
\text { No visible wrinkles; continuous skin line }\end{array}$ \\
\hline 1 (mild) & $\begin{array}{l}\text { Fine wrinkles } \\
\text { Visible wrinkles and slight indentation }\end{array}$ \\
\hline 2 (moderate) & $\begin{array}{l}\text { Moderate wrinkles } \\
\text { Cleary visible wrinkles }\end{array}$ \\
\hline 3 (severe) & $\begin{array}{l}\text { Deep wrinkles } \\
\text { Deep and furrowed wrinkles }\end{array}$ \\
\hline 4 (extreme) & $\begin{array}{l}\text { Extremely deep wrinkles } \\
\text { Extremely deep and long folds }\end{array}$ \\
\hline
\end{tabular}

Table 2. Global Aesthetic Improvement Scale (GAIS)

\begin{tabular}{ll}
\hline \multicolumn{1}{c}{ Rating } & \multicolumn{1}{c}{ Description } \\
\hline Worse & Appearance worse than baseline \\
No change & Appearance same as baseline \\
Improved & $\begin{array}{l}\text { Obvious improvement in appearance from } \\
\text { initial condition }\end{array}$ \\
Much improved & $\begin{array}{c}\text { Marked improvement in appearance from } \\
\text { baseline, but not completely optimal }\end{array}$ \\
Very much improved & Optimal cosmetic result \\
\hline
\end{tabular}



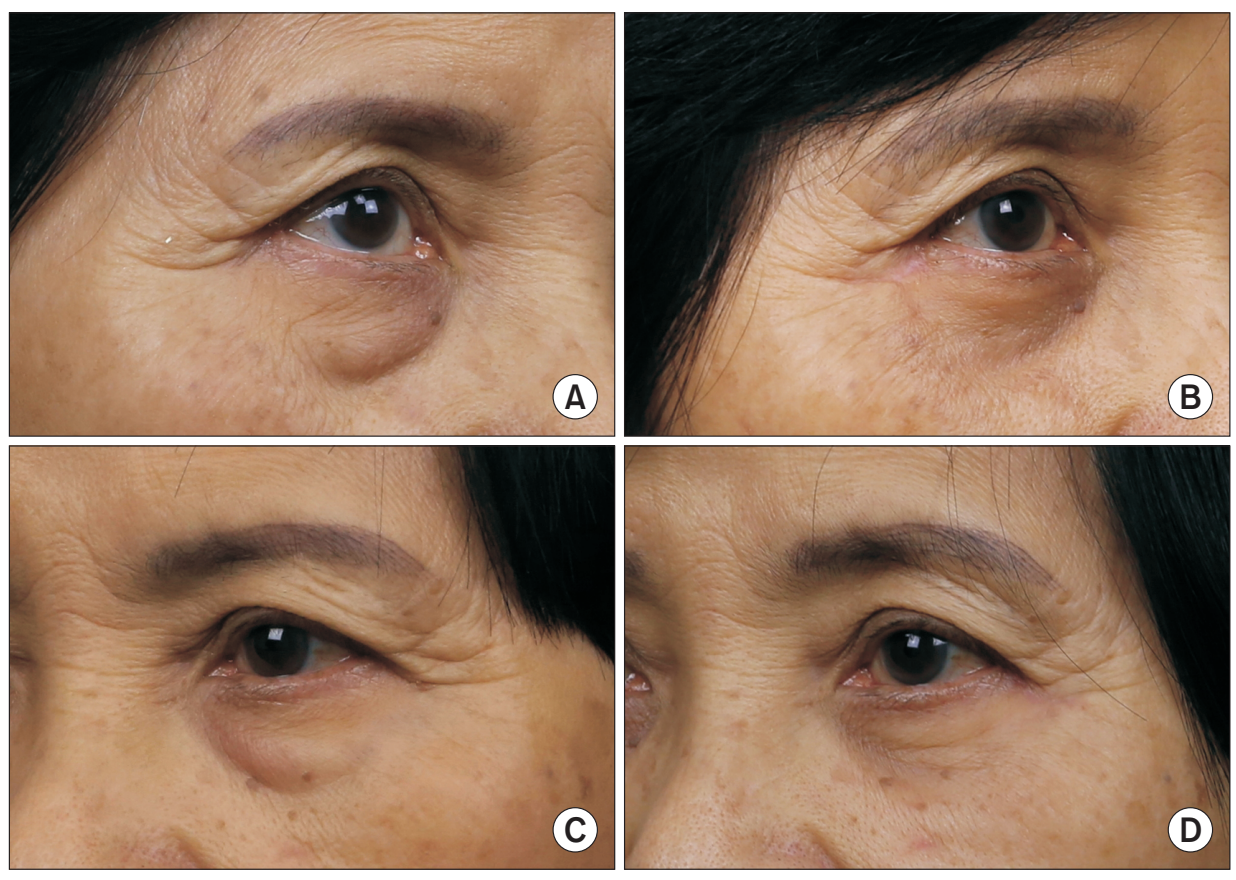

Fig. 4. Before and 2 months after treatment photos. (A) Before in RF group, (B) after in RF group, (C) before in LB group, (D) after in LB group. RF group, microneedle radiofrequency device group; LB group, lower blepharoplasty alone group.

Table 3. Comparison of Wrinkle Assessment Scale (WAS) in before and 2 months after treatment

\begin{tabular}{lcc}
\hline $\begin{array}{c}\text { Changes in WAS } \\
\text { (2 months post-operation) }\end{array}$ & RF group & LB group \\
\hline Improvement & $9(75.0)$ & $7(58.3)$ \\
No change & $3(25.0)$ & $5(41.7)$ \\
Worsening & 0 & 0 \\
\hline
\end{tabular}

Values are presented as $\mathrm{n}(\%)$.

$\mathrm{RF}$ group, microneedle radiofrequency device group; LB group, lower blepharoplasty alone group.

an average age of 58 years (range, $42-74$ years). Three of them were Fitzpatrick skin type III, six were type IV, and three were type V. All patients attended follow-up visits until 6 months from the first procedure, and all were satisfied with the results. At the 2-month postoperative follow-up, all the patients chose to undergo microneedle RF treatment for the other eye. The pre- and postoperative average WAS scores were 2.33 and 1.5, respectively, for the RF group, and 2.0 and 1.42, respectively, for the LB group. In the RF group, $75.0 \%$ (9 out of 12 eyes), and in the LB group, $58.3 \%$ ( 7 out of 12 eyes), showed improvement in wrinkles (Fig. 4). Three eyes in the RF group and five eyes in the LB group showed no changes in the WAS score. No patient showed worsening of wrinkles in either group (Table 3). The wrinkle reduction between the RF and LB groups was statistically insignificant $(\mathrm{p}=0.317)$. The postoperative inflammation difference between the RF and LB groups was not noticeable at 1 week postoperatively.

The patient self-evaluation using GAIS is shown in Fig. 5. All

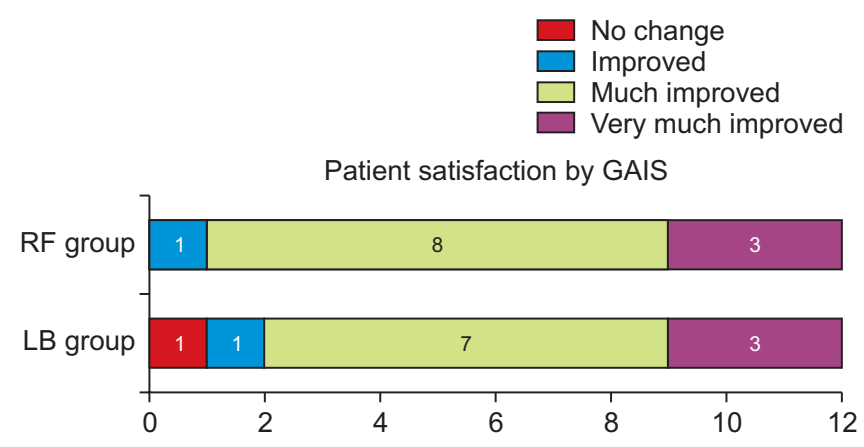

Fig. 5. Patient satisfaction evaluated by Global Aesthetic Improvement Scale (GAIS). RF group, microneedle radiofrequency device group; LB group, lower blepharoplasty alone group.

patients in the RF group eye experienced improvement in their wrinkles in the lower eyelid area. One patient graded herself as having no improvement in the LB eye. However, GAIS showed high patient satisfaction in both groups, with an average of 4.12 (3-5) in the RF group and 4.0 (2-5) in the LB group ( $\mathrm{p}=0.157)$. In the RF group, none of the patients felt discomfort while undergoing microneedle RF treatment, although there was some pinpoint redness at the microneedle entry sites in the first 2-3 days, which spontaneously improved. No persistent erythema was noted after 1 week. No PIH occurred during the 6-month follow-up period. No other adverse effects were observed.

\section{Discussion}

In recent years, it has been generally accepted that lower eye- 
lid rejuvenation should be combined with lower blepharoplasty in skin tightening and wrinkle reduction procedures. One of the most popular procedures frequently performed with lower blepharoplasty is ablative resurfacing lasers, such as $\mathrm{CO}_{2}$ and erbium yttrium aluminum garnet lasers. Unquestionably, the $\mathrm{CO}_{2}$ resurfacing laser is powerful in fine wrinkle reduction and has been widely used for the treatment of periorbital wrinkles $[3,8]$. Because of the high prevalence of PIH after treatment, especially in Asians with darker skin, $\mathrm{CO}_{2}$ lasers may not be safe for use. Consequently, insulated microneedle RF devices have been developed in Korea as an alternative treatment for periorbital wrinkle reduction in Asians. Microneedle RF has less downtime when compared to an ablative resurfacing laser because there is no wound at the skin epithelium. In this study, we proved that the microneedle RF device can be safely applied at the conclusion of transconjunctival lower blepharoplasty.

In a previous study, Lee et al. [6] treated periorbital wrinkles with a fractional microneedle RF device in three sessions at 4 -week intervals. The treatment parameters were needle insertion to $0.8 \mathrm{~mm}$ depth, with a power of $12.5 \mathrm{Watt}$ and duration of $100 \mathrm{~ms}$. They demonstrated that $90 \%$ of the patients were satisfied with wrinkle reduction and reported a lower rate of PIH when applied in dark-skinned patients. This study revealed that $75 \%$ of patients who underwent lower blepharoplasty combined with one session of microneedle RF experienced wrinkle improvement. Similar to most skin rejuvenation devices, multiple applications are recommended by the manufacturer to achieve noticeable results. The major drawback of the microneedle RF includes multiple skin punctures per session, which require local anesthetic injection because topical anesthetic medication may not be sufficient. Performing lower blepharoplasty and microneedle RF simultaneously reduces the procedure session and decreases the total recovery period.

In this study, there was no statistically significant difference in wrinkle reduction between the RF group eyes and LB group eyes. The LB group eyes showed $58.3 \%$ improvement in wrinkles, and no eye showed a worsening of wrinkles as assessed by the WAS. Carter et al. [3] reported that $46 \%$ of Caucasians experienced a worsening of wrinkles after undergoing transconjunctival lower blepharoplasty alone. Our distinctive results could be due to two reasons. First, we performed orbicularis oculi suspension and skin removal at the lateral canthus area before closing the lateral canthus incision. Second, Asian eyelid skin possesses a thicker dermis and fewer wrinkles than that of Caucasians. Nevertheless, the trend of greater wrinkle improvement shifted toward the RF group eye. The sample size calcula- tion to find statistically significant results of this study was based on a previous study in Caucasians, which provided less than an adequate number of samples.

The GAIS showed high average scores in both the RF and LB groups. There was also no worsening of wrinkles according to patient self-assessment. One patient felt no wrinkle improvement in her LB group eye, whereas all of the patients were satisfied with the wrinkle improvement in the RF group eye. Moreover, all of the patients were willing to undergo microneedle $\mathrm{RF}$ for the other eye at the 2-month follow-up visit, which indicated that they all felt their RF group eye had superior results.

None of the patients in our study experienced microneedle $\mathrm{RF}$ device-related complications and other postoperative lower blepharoplasty complications after a 6-month follow-up period. Another microneedle RF advantage is a low rate of $\mathrm{PIH}$, unlike the ablative resurfacing laser report [9].

In conclusion, transconjunctival lower blepharoplasty with or without microneedle RF treatment produced wrinkle improvement. The insulated microneedle RF device proved to be safe when performed concurrently with lower blepharoplasty.

\section{Conflicts of interest}

The authors have nothing to disclose.

\section{References}

1. McCann JD, Pariseau B. Lower eyelid and midface rejuvenation. Facial Plast Surg 2013;29:273-80.

2. Hidalgo DA. An integrated approach to lower blepharoplasty. Plast Reconstr Surg 2011;127:386-95.

3. Carter SR, Seiff SR, Choo PH, Vallabhanath P. Lower eyelid $\mathrm{CO}(2)$ laser rejuvenation: a randomized, prospective clinical study. Ophthalmology 2001;108:437-41.

4. Manuskiatti W, Siriphukpong S, Varothai S, Wanitphakdeedecha R, Fitzpatrick RE. Effect of pulse width of a variable square pulse (VSP) erbium:YAG laser on the treatment outcome of periorbital wrinkles in Asians. Int J Dermatol 2010;49:200-6.

5. Greene RM, Green JB. Skin tightening technologies. Facial Plast Surg 2014;30:62-7.

6. Lee SJ, Kim JI, Yang YJ, Nam JH, Kim WS. Treatment of periorbital wrinkles with a novel fractional radiofrequency microneedle system in dark-skinned patients. Dermatol Surg 2015;41:615-22.

7. Calderhead RG, Goo BL, Lauro F, Gursoy D, Savant S, Wron- 
ski A. The clinical efficacy and safety of microneedling fractional radiofrequency in the treatment of facial wrinkles: a multicenter study with the INFINI system in 499 patients [Internet]. Goyang: Lutronic; c2013 [cited 2017 May 28]. Available from: http://ayalasers.com/pdf/INFINI_WP_multicenter_499_cases_20130710.pdf.

8. Roberts TL 3rd, Yokoo KM. In pursuit of optimal periorbital rejuvenation: laser resurfacing with or without blepharoplasty and brow lift. Aesthet Surg J 1998;18:321-32.

9. Chan HH, Manstein D, Yu CS, Shek S, Kono T, Wei WI. The prevalence and risk factors of post-inflammatory hyperpigmentation after fractional resurfacing in Asians. Lasers Surg Med 2007;39:381-5. 